\title{
Faits et fictions géographiques dans le roman régional suisse: Le cas de Via Mala
}

\author{
«Le col de l'Yzolla n'est praticable que durant les quatre mois d'été; le reste du temps il est enfoui \\ sous la neige depuis la chapelle Saint-Hubert sur le versant suisse jusqu'aux précipices du Sud qui \\ descendent sur la Valteline. La route d'Andruss à l'auberge d'Yzolla s'appelle la Via Mala.»
}

John Knittel, Via Mala (1939)

JoHN KNITTEl (1891-1970) fut un grand écrivain suisse qui explora des styles et des procédés littéraires uniques. Une partie de son œuvre se déroule dans les Grisons en des lieux et paysages dont il convient de fixer la dose de réalité et de fiction. Ce cadre géographique touche notamment Via Mala, son best-seller et son seul roman à connotation helvétique. C'est donc dans le cadre grandiose des sommets et des vallées de la Surselva et de l'Oberhalbstein que se passe l'action dramatique de cette œuvre à laquelle KNittel dut le renom international.

KNitTel a dépeint le décor géographique de Via Mala avec une telle consistance que, pour beaucoup de lecteurs, il ressemble à un territoire réel. Chez KNitTel, la fiction ou la réalité modifiée influencent profondément la perception que le lecteur possède de ces lieux. Toutefois il existe des questions intrigantes entre la Via Mala réelle et celle de Knittel. Cette dernière ne serait-elle pas la description d'autres lieux et d'autres paysages maquillés sous ce nom? Ainsi, beaucoup d'aspects géographiques dans cette grande œuvre suisse méritent d'être considérés et les processus par lesquels les faits géographiques sont transformés en fictions se doivent d'être synthétisés. L'analyse proposée ici cherchera donc à identifier les aspects reconnus de la réalité géographique concernée. Elle ira aussi au-delà de la géographie ordinaire des Grisons en mettant en relief des matériaux géographiques de base que peu de personnes invoquent.

Pour bien interpréter géographiquement la Via Mala de Knittel, il est important de réaliser que le roman a été écrit entre les deux guerres mondiales à une époque où la modernisation n'avait pas complètement et profondément pénétré les hautes vallées des Grisons. Knittel, né en 1891 aux Indes, d'un père pasteur protestant, est retourné en Suisse dès 1896 et c'est en avril 1970 qu'il mourut à Maienfeld. Des centres comme Coire, Disentis, Lenzerheide et SaintMoritz n'avaient pas, à cette époque, atteint le développement économique et l'extension spatiale qu'ils connaissent aujourd'hui. Il faut aussi ne pas perdre de vue qu'au moment de la rédaction de Via Mala, une bonne partie des communications locales s'effectuait encore en charrettes à cheval.

De ce fait, les mini-régions ou les vallées montagnardes servant de support aux territoires évoqués dans Via Mala avaient un profil assez différent de celui que nous leur connaissons aujourd'hui et, qui plus est, elles étaient passablement isolées. Cependant, la vallée du Rhin antérieur, le Val Medel, la contrée de Coire et l'Oberhalbstein, rendus par KNitTel, traduisent fort bien la diversité physique rencontrée sur le terrain. Même si la morphologie urbaine et rurale a quantitativement changé depuis 1939, elle demeure la même dans ses fondements et les contrées grisonnes concernées par Via Mala desservent toujours une société agraire et touristique.

\section{Val Medel et Surselva: les faits à la base de la fiction}

Par une série convergente de noms géographiques réels, il est possible de fixer le cadre spatial détaillé du territoire véhiculé dans Via Mala. L'action romanesque impliquant les deux héros de l'œuvre, Sylvia Lauretz et ANDRÉas de Richenau, se déroule en quatre endroits différents: le Val Medel, l'Oberland grison ou Surselva, la région de Coire et l'Oberhalbstein. Il convient de reprendre successivement chacune de ces quatre régions pour bien saisir l'intelligence des rapports entre la géographie réelle et la géographie revue et corrigée par KNITTEL.

Certains toponymes et descriptions disposés dans le roman permettent d'affirmer que le cœur de l'action se déroule dans le Val Medel que Knittel appelle, pour son usage littéraire, la Via Mala. Il prend même

*Docteur d'Etat. Auteur d'une thèse sur "La Suisse, essai de géographie politique" (Paris IV). Professeur, Université du Québec, Chicoutimi G7H 2B1, Canada.

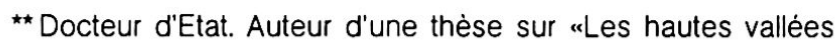
du Rhin (Grisons)" (Grenoble I). Maître-assistant, Université de Grenoble I, Institut de Géographie Alpine, 38031 Grenoble, France. 


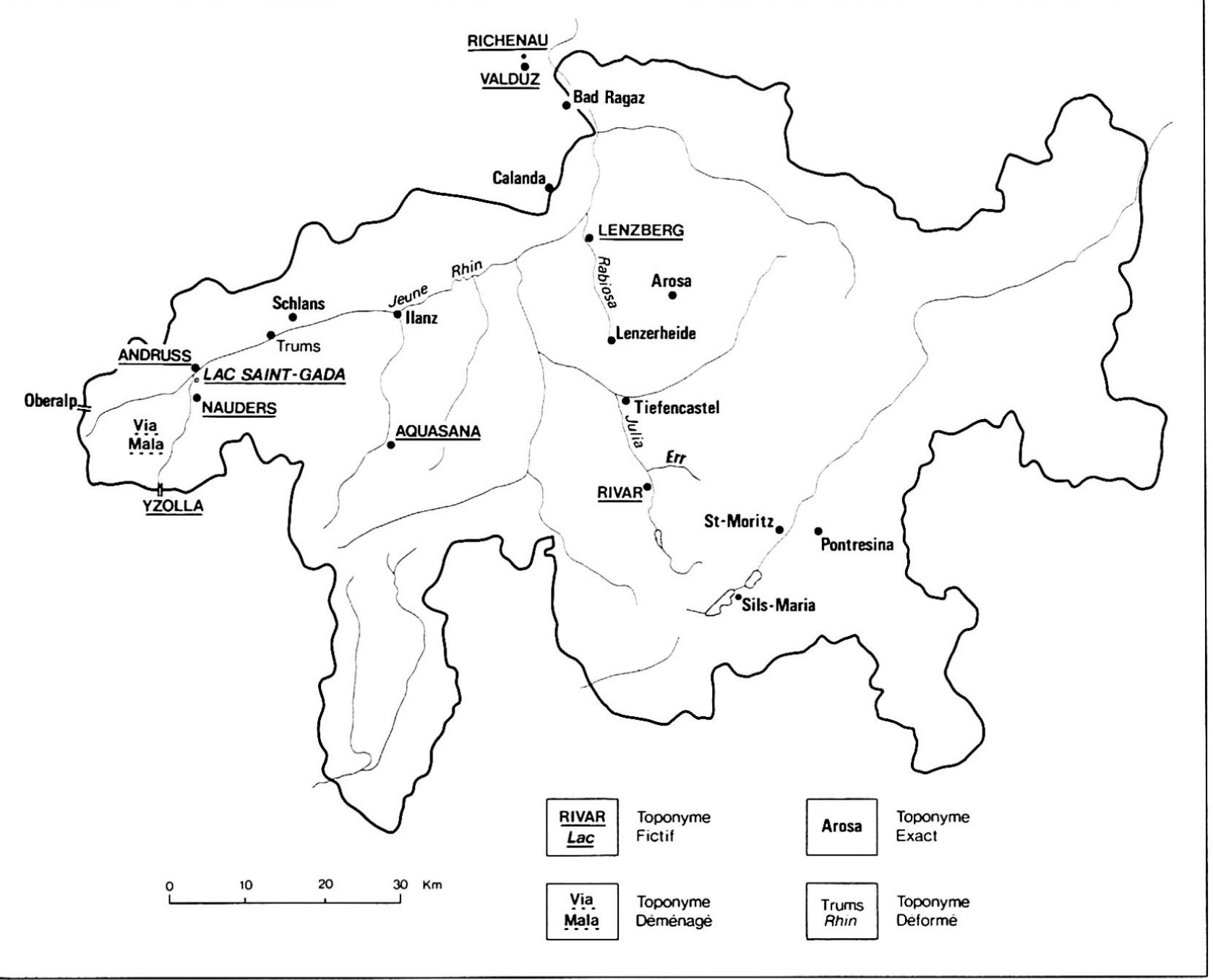

Fig. 1 Les Grisons revisités par Knittel: un processus classique de conversion littéraire

le soin de préciser que sa Via Mala constituant la vallée de l'Yzolla est «une étroite vallée latérale du Haut-Rhin» (p.7). Au début du roman, l'auteur évoque les minerais argentifères à proximité de la vallée (p.9, 10). Cette particularité géologique se rencontre effectivement dans le Val Cristallina, affluent de rive droite du Rhin de Medel. Dans cette introduction où il plante le décor, KNitTel parle des «eaux mugissantes de la cascade» (p.7) se précipitant d'une hauteur de trente mètres au fond d'un ravin (p. 23). Cette cascade, c'est la Fumatschfall, en plein cœur du Val Medel. Toujours dans ce registre des repères topographiques naturels, plusieurs noms de sommets locaux réels sont très souvent identifiés. En maints endroits, il est question du Pic Cristallina $(3128 \mathrm{~m})$ dont KNITTEL développe la spécificité: parois abruptes, épaule de glace (p. 30, 49, 122). Il mentionne également d'autres sommets faisant partie de la même chaîne: Pic Rondadura (3016 m), à l'Ouest immédiat du Lukmanier, à l'orthographe escamotée en Pic Ronda (p.49), Pic Vallatscha (3109 m), à l'Est du Lukmanier, ce nez rocheux regardant la Via Mala et transcrit par KNitTel sous le vocable de Valatch (p. 58). Le lecteur retrouve aussi dans le texte le glacier de Vial (p. 122), le flanc Nord du Pic Valdraus $(3096 \mathrm{~m})$ et son sommet neigeux (p. 308, 310), le Pic Uffiern $(3151 \mathrm{~m})$, modifié en Ufiern par KNITTEL, et qu'on aperçoit derrière Cristallina sans oublier tout à côté le Pic Medel (p. 322).

$A$ ces repères physiques viennent s'ajouter des localisations humaines tout aussi exactes. Dans ce Val Medel maquillé en Via Mala, la réalité géographique finit par transpercer. A plusieurs reprises, le lieu-dit Santa Maria apparaît (p. 10,51) et KNitTel indique même que cet endroit se localise au pied du col et se trouve enfoui sous la neige plusieurs mois par an. Effectivement, Santa Maria existe et a donné son nom au lac artificiel de retenue situé au pied du Lukmanier sur versant rhénan. Tout à côté, en manipulant l'orthographe en Saint-Gion (p. 10) ou en San Gian (p. 477), le romancier fait surgir le hameau de Sogn Gions dominant les profondeurs sauvages de la vallée. En évoquant le versant Sud du col, KNitTel parle aussi des cabaretiers de ponte Valentino, Castro et 


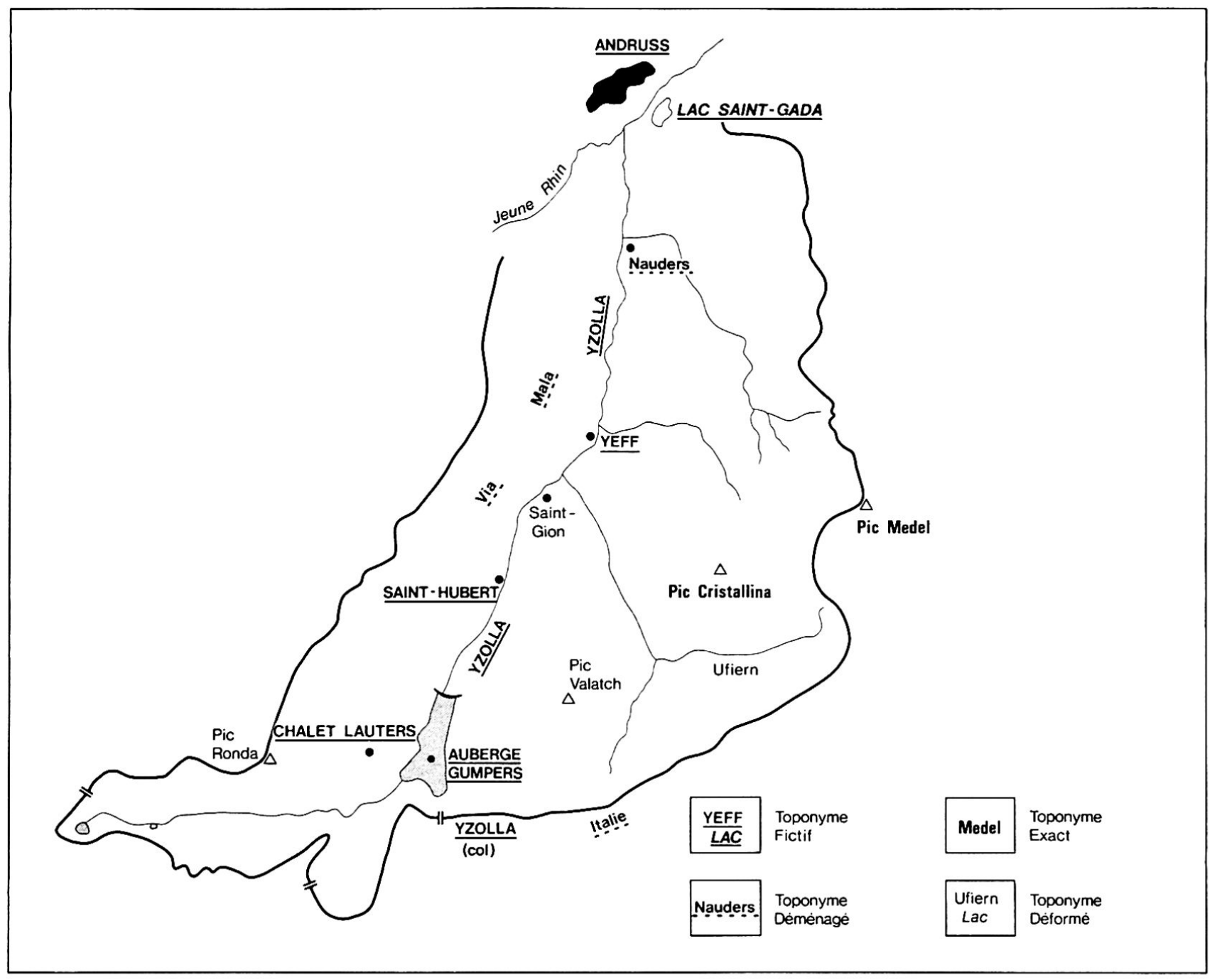

Fig. 2 Val Medel/Via Mala: une géographie revue et corrigée par Knittel

Biaska (Biasca). Ces lieux sont exacts puisqu'il s'agit de trois villages ou bourgs du Val Blenio en Tessin (p. 10). Au chapitre XVII, le père de Sylvia, Jonas LAURETZ, suit en carriole la route de Medels (orthographe exacte: Medel) montant par une trouée, avec le jeune Rhin coulant en contrebas (Rhin de Medel). En quittant Disentis pour monter vers la Via Mala et le col de l'Yzolla, il faut franchir un pont suspendu audessus du Rhin puis passer à travers des tunnels (p.430). C'est la Medelserschlucht dans la partie inférieure du Val Medel, peu en amont de la confluence entre Rhin de Medel et Rhin antérieur (lieudit Fontanivas). Enfin, lorsque le vieux Lauretz remonte chez-lui dans la vallée de l'Yzolla il atteint la fourche de la route: à gauche, c'est la Via Mala, à droite la route du Val Tavetsch (p. 73, 74).

A ce stade de l'analyse, une partie du cadre environnemental commence à prendre une vigueur insoupçonnée. Les indices tant physiques qu'humains situent bien la Via Mala comme étant le Val Medel. Il est nécessaire toutefois de décortiquer géographiquement la deuxième région du roman de KNITtel, la Surselva ou vallée du Rhin antérieur. Explicitement, l'action romanesque centrée sur la Via Mala se greffe et s'appuie sur la Surselva. Là encore, beaucoup de lieux et de paysages réels aident à démasquer l'écran littéraire. En termes de traits physiques, KNitTel mentionne le col de l'Oberalp (p. 12) en détaillant avec exactitude que le soleil s'y couche derrière (c'est la direction franc Ouest). Plus loin, le lecteur lit: «Les derniers nuages lumineux au-dessus de la chaîne de l'Alpetta se reflétaient dans un lac sombre et rond...» (p. 73). En effet, le Pic Alpetta $(2764 \mathrm{~m})$ se localise au Nord-Est immédiat de Disentis et le lac en question peut s'identifier comme étant le lac de Laus. En d'autres sections du roman apparaissent des toponymes fort connus dans la Surselva: Tavetsch (p. 28), d'ailleurs romanchisé en Tujetsch depuis mai 1976, où les Gumpers, propriétaires de l'auberge du col de l'Yzolla, passent tous les hivers (p. 51); Sedrun (p. 33), fraction de la commune de Tavetsch..

A l'Est de Disentis, dans la partie aval du Rhin antérieur, d'autres toponymes réels permettent de cerner petit à petit le décor: Schlans où le jeune couple 
Richenau s'est installé dans une ferme donnée par le père d'ANDRÉAS. Là, KNITTEL démontre parfaitement sa capacité à restituer avec beaucoup de finesse le cadrage géographique: «Le petit village de Schlans était situé sur le côté ensoleillé d'une très large vallée verdoyante, pas très loin de la grande route de l'Oberland» (p. 364). De fait, Schlans s'étale sur le versant en adret de la Surselva, c'est-à-dire sur le versant orienté plein Sud et la route 19 Coire-Oberalp passe en contrebas, le long du Rhin, à 500 mètres du village. Quelques kilomètres plus en aval, en direction de Coire, KNittel fait également évoluer ses personnages à Ilanz avec ses auberges et ses scieries (p.48, 179).

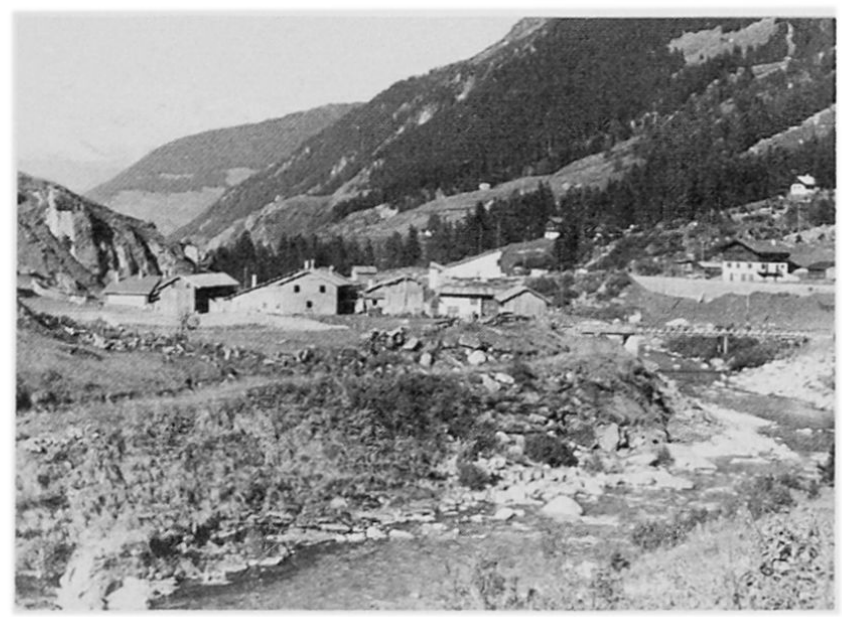

Fig. 3 Imyeff et la vallèe de I'Yzolla

"Dans une étroite vallée latérale du Haut-Rhin la vallée de I'Yzolla, une maison isolée se dressait à côté d'une scierie. On l'appelait la maison Imyeff;... elle était habitée par la famille Lauretz... et était située à une centaine de mètres de la grande route,... sous la muraille grise et nue d'une montagne qui la surplombait... de sept cents mètres."

Sur le cliché (1981) on reconnait le décor décrit par J. Knittel: la vallée du Rhin de Medel (I'Yzolla) vers l'aval. A gauche, le hameau d'Acla (Yeff) au bas d'une muraille de granite gris à l'écart de la route qui relie Disentis (Andruss) au Lukmanier. Aucune trace de la scierie de J. Lauretz; quant à la maison décrite par Knittel, il pourrait bien s'agir de la troisième en partant de la gauche.

\section{La région de Coire et l'Oberhalbstein: la réalité comme support de l'imagination}

Sylvia LAURETZ et ANDRÉAs de Richenau, partenaires centraux du roman, évoluent en certains épisodes dans ces deux contrées grisonnes. Sous le pseudonyme de Lanzberg, Coire ressort très nettement dans toute sa spécificité. «A Lanzberg, convergeaient toutes les anciennes routes que les peuples migrateurs parcouraient du Nord au Sud et vice versa depuis des siècles. Pour atteindre les cols de Splügen, de Maloja, du Bernardino, de l'Oberalp et de l'Yzolla, il fallait passer par Lanzberg» (p. 193). L'écrivain ajoute d'autres détails qui font sauter les dernières hésitations: la prison cantonale (p. 109), l'avenue de la Gare, la partie moyenâgeuse de la ville, la cathédrale, l'évêché, le siège du gouvernement du canton (p. 194, 195), l'important centre ferroviaire, la gare des CFF (p. 193). Enfin, KNitTel précise le tableau par une signature topographique indéniable: "Juste derrière la ville, s'élevaient les larges épaules du Calanda, le Mitterberg abrupt et la rocheuse Platte» (p. 194, 195). Le massif de Calanda domine Coire au Nord-Ouest à $2805 \mathrm{~m}$ d'altitude, tandis que le Mittenberg (orthographe exacte) et Rot Platte $(1505 \mathrm{~m})$, constituent un contrefort surplombant l'Est immédiat de la ville. De Lanzberg, le couple ANDRÉAS-SyLviA monte faire une promenade en voiture vers Lenzerheide (p. 265) en longeant la Rabiosa (Rabiusa) pour prendre un bain dans le Heidsee (p. 270-271). Non seulement, l'itinéraire et les lieux sont véridiques mais encore une baignade publique existe bel et bien au Heidsee à l'entrée Nord de Lenzerheide.

Enfin, un autre volet de l'action romanesque se déroule dans l'Oberhalbstein, c'est-à-dire dans la vallée de la Julia ou Gelgia, entre Tiefencastel et le Julierpass. Andi (ANDrÉas) emmène Sylvelie (SYlvIA) dans un chalet de famille sur l'Alpe d'Err (p.340, 343). Ce lieu est réel. Il est situé à 2177 mètres dans la partie amont du Val d'Err qui conflue avec la Julia à Tinizong. KnitTel parle également de la «vieille ferme de famille à Rivar» près de laquelle la Julia coule comme du cristal liquide (p. 223). Non loin de la confluence Val d'Err-Julia, cet endroit, c'est Rieven, fraction de la commune de Rona.

\section{La fiction comme mutation des faits}

Les comparaisons géographiques entre le réel et l'imaginaire sont frappantes dans le roman de KNITTEL. Comme Disentis, Andruss est la ville principale de la "vallée du jeune Rhin". Comme Coire, Lanzberg est une ville-carrefour aux fonctions tertiaires diversifiées. Comme Disentis et Coire, Andruss et Lanzberg sont desservies par la voie ferrée. Comme la Surselva-Oberland, l'espace de KNitTel possède ses sous-régions, ses lieux distinctifs et ses repères. Malgré sa fiction, KnitTel n'a aucun doute sur la localisation exacte de sa Via Mala. La situation de Via Mala est plausible au niveau des concepts de perception. KNitTel n'a jamais publié une carte détaillée d'Andruss mais d'importantes similitudes entre cette ville fictive et Disentis sont aisément déchiffrables. Le monastère bénédictin d'Andruss, c'est celui de Disentis (p. 8, 9), effectivement incendié par les troupes françaises en 1799. Le cabaret «A la Vieille Poste» au cœur d'Andruss, c'est la vieille et historique Stiva Grischuna au centre de Disentis (p.12). La fausse 
Andruss dispose de son journal intitulé «Le Courrier» (p.69), c'est la Gasetta Romontscha de Disentis. A proximité d'Andruss, le nouveau pont de Larchestutz (p. 26), c'est le punt Russein, non loin de la confluence du Val Russein et du Rhin antérieur entre Disentis et Somvix.

Même si beaucoup d'analogies persistent entre Andruss et Disentis, entre l'espace knittelien de Via Mala et les hautes vallées du Rhin, un examen minutieux et serré révèle de profondes distorsions entre le réel et le fictif. Ainsi, il n'y a jamais eu de lac Saint-Gada (p.271, 354) à proximité de Disentis. KNITTEL invente totalement. Tout au plus, au pied de Disentis, sur le Rhin, se localise une chapelle nommée Sontga Gada. Nandero (p. 10), quelque part entre Disentis et le Val Blenio au Tessin, est un lieu purement imaginaire que KNITTEL crée en empruntant par déformation le toponyme Val Nandró, affluent de la Julia à Savognin. Même constatation pour Aquasana (p. 492), lieu totalement inconnu aux Grisons. Aquasana (eau saine) peut constituer une transcription fantaisiste de Bad Ragaz ou de Vals, toutes deux stations thermales.

Il est fort douteux qu'il y ait eu des cabaretiers à Sogn Gions et à Santa Maria sur la route du Lukmanier (p. 10). En effet, ces lieux-dits sont minuscules et, qui plus est, enfouis sous la neige et inaccessibles pendant la moitié de l'année. Dès lors, la Via Mala de ЈонN KNittel est une composition de lieux actuels mais des mutations fictives, au sein d'éléments géographiques tirés de la réalité, altèrent délibérément cette dernière. Il convient donc d'analyser ces processus de sublimation de la géographie actuelle.

\section{Les processus de conversion chez Knittel}

Knittel a transmuté le Val Medel en Via Mala en combinant le réel, le transformé et l'imaginaire. La géographie de la Surselva et de la commune de Medel-Lucmagn est changée au moyen de quatre principaux outils: l'altération des toponymes, l'omission des composants, le glissement spatial des localisations et, enfin, le mélange du réel avec le fabriqué (Figure 1).

L'altération des toponymes et, plus précisément le changement de noms, est la première technique utilisée par KNITTEL. D'une part, l'écrivain transforme carrément l'étiquette toponymique: Andruss à la place de Disentis, Lanzberg remplaçant Coire, Larchestutz pour Russein, Rieven changé en Rivar... D'autre part, l'escamotage orthographique est une méthode permettant de mystifier le lecteur: Mitterberg pour Mittenberg, Platte au lieu de Rot Platte, Trun dévié en Trums (p. 492), Rabiosa au lieu de Rabiusa, Biaska pour Biasca, Sogn Gions devenu San Gian ou Saint-Gion, Ufiern pour Uffiern, Pic Ronda et Pic Valatch remplaçant Rondadura et Vallatscha...

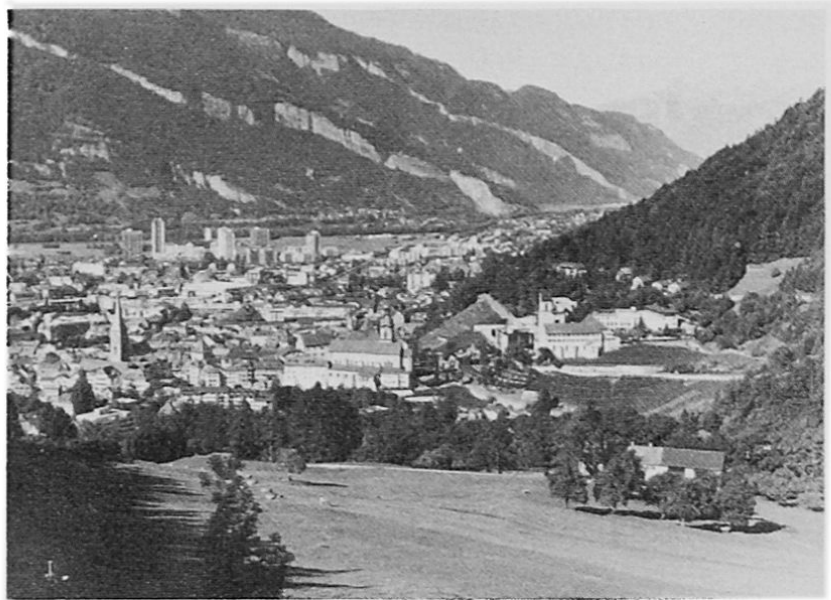

Fig. 4 Lanzberg

"D'un seul regard sur la ville, on comprenait que les quinze mille habitants de Lanzberg n'avaient pas connu la pauvreté depuis bien des années. La prospérité était devenue habituelle chez eux et comme c'est le cas pour la plupart des cités florissantes, la situation géographique de Lanzberg y était pour beaucoup."

Le Lanzberg de Knittel, c'est Coire, la capitale du canton des Grisons (33000 habitants aujourd'hui). Le cliché, pris en direction du Rheintal, montre une grande partie de la ville avec son extension récente sous forme de "tours". Au premier plan, la vieille ville, bâtie autour de la cathédrale.

L'omission des détails représente une seconde technique simple par laquelle KNITTEL altère la géographie des hautes vallées du Rhin antérieur. Via Mala est un modèle et, malgré ses complexités, elle est, comme tous les modèles, moins abstruse que la réalité. Si toutes les descriptions géographiques et les toponymes de KNitTel étaient ajustés ensemble pour créer une carte totale de l'espace Via Mala, cette carte serait plus petite que la carte du Val Medel tout entier. KNITTEL omet délibérément des parties essentielles des Grisons occidentaux. Flims et d'autres petits bourgs de la Surselva sont exclus de Via Mala. Tinizong, Savognin et Marmorera sont gommés de l'Oberhalbstein tandis qu'Arosa, Lenzerheide et Tiefencastel ne sont que rapidement mentionnés. En somme, la Via Mala apocryphe de KNitTel ne contient ni les détails ni la totalité de l'espace dont elle est la sublimation littéraire.

Le glissement spatial des localisations constitue une troisième technique employée par KNitTel. Ce glissement s'effectue à des échelles soit locales, soit régionales, soit inter-régionales. A l'échelon local, le meilleur exemple est celui fourni par ANDi et SylveLIE, au sommet du Pic Julier, contemplant à leurs pieds les lacs de l'Engadine et le village de Sils-Maria (p. 343). Le panorama est quelque peu distordu car, entre le Pic Julier et Sils-Maria, vient s'interposer la masse du Pic Polaschin $(3013 \mathrm{~m})$. De même, un simple examen de la carte topographique ne peut 
permettre d'affirmer que le Pic Julier (3380 m) est «au même niveau» que le Pic Morteratsch (3751 m).

A l'échelon régional, quelques illustrations peuvent également être fournies. La véritable Via Mala constitue le défilé fameux du Rhin postérieur entre Thusis et Andeer. Par un procédé fort habile, KNITTEL fait glisser ce toponyme cinquante kilomètres plus à l'Ouest pour l'appliquer au Val Medel et ainsi égarer le lecteur. De la même manière, ses toponymes apocryphes d'Andruss et de Yeff peuvent faire penser qu'il s'agit d'Andeer et de Juf. Le procédé est subtil! Cette méthode knittelienne de glissement spatio-temporel est très bien démontrée par le cas de Schlans dans l'Oberland grison. L'écrivain explique que le jeune couple Richenau habite à Schlans et que le mari va travailler à Coire tous les jours. Selon KNITTEL, Schlans est à vingt minutes de voiture de la capitale cantonale (p.338). Cela est impossible pour deux raisons. Premièrement, le roman a été écrit en 1939 et les communications routières ainsi que les automobiles n'étaient pas ce qu'elles sont aujourd'hui. Deuxièmement, la route, qui de Coire monte à Disentis, est parfois tortueuse; et surtout, il y a une distance de 47 kilomètres entre Schlans et Coire.

A l'échelon inter-régional, Via Mala offre encore d'autres excellents exemples. En appliquant au Lukmanier l'appellation fictive de col de l'Yzolla, KNITTEL place un écran trompeur entre la réalité et son propre espace littéraire en empruntant à trois noms de lieux réels: Isola est le nom d'un petit lac au pied du village de San Bernardino mais Isola, c'est aussi un lieu-dit entre le Splügenpass et Campodolcino en Valteline et c'est, enfin, un autre lieu-dit sur la berge Sud du lac de Sils en Haute-Engadine. Ce glissement spatio-temporel à l'échelle inter-régionale ressort fortement dans les cas de Valduz et de Richenau. La technique de KNITTEL consiste à laisser plus ou moins croire au lecteur que Valduz, c'est Vaduz (Liechtenstein), petite ville dominée par le château princier transformé ici en château de la famille Richenau. Knittel transporte au-dessus de Valduz le château réel de Reichenau situé à la confluence exacte du Rhin postérieur et du Rhin antérieur, à une dizaine de kilomètres au Sud-Ouest de Coire. Toutefois, les descriptions environnementales précises qu'apporte KNITTEL permettent d'affirmer que Valduz, c'est Sargans et que le château Richenau dominant la ville, c'est celui de Sargans au Nord immédiat de la ville (p. 220, 235). Par glissement chronologique (p.53), l'écrivain indique que Valduz (Sargans) est à une heure de voiture de Lanzberg (Coire). Même en 1939, le rapport temps-distance était surévalué pour la bonne raison que, par la route cantonale classique, la distance entre les deux villes s'établit à 30 kilomètres! La combinaison de la fabrication avec les faits constitue, finalement, la quatrième technique appliquée par KNITTEL dans ses processus d'altération de la réalité géographique. JoHN KNITTEL brosse dans une description convergente les quatre sommets voisins de

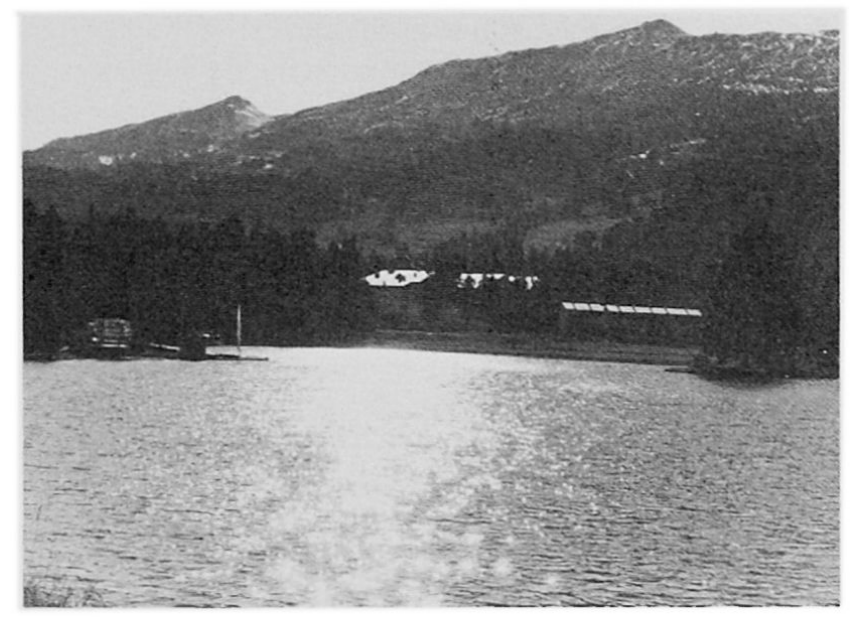

Fig. 5 Le Heidsee

"A travers une clairière, un lac bleu scintillait comme un champ de turquoise. Andi s'ariêta.

- Heidsee, s'ècria-t-il, en baissant la vitre de la portière. Si on se baignait?"

Le Heidsee est aujourd'hui la baignade de la station de Lenzerheide/Lai, un des joyaux des Grisons, sur un long col à 1100 mètres d'altitude. Les activités nautiques concernent actuellement le canotage et le windsurfing. En hiver le lac, gelé, sert de vaste patinoire naturelle.

Cristallina, Uffiern, Medel et Valdraus dominant le Val Medel (p. 310, 322). Du chalet Lauters situé à proximité du col de l'Yzolla, l'écrivain fabrique avec le réel en permutant la position exacte des sommets: Cristallina et Uffiern sont placés derrière Medel et Valdraus alors que, sur le terrain, ils se situent entre le Lukmanier et Medel-Valdraus. De même, KNitTel fait croire à ses lecteurs que le col de l'Yzolla forme frontière entre la Suisse et l'Italie. Par là, il emprunte les connotations politico-territoriales du Splügen voisin ou de l'Umbrailpass plus lointain. En réalité, le Lukmanier est seulement frontière inter-cantonale avec le Tessin (p.357, 379). En somme, l'italianité culturelle tessinoise est sublimée en italianité politique!

\section{Val Medel/Via Mala: archetype d'un processus de conversion littéraire}

En elle-même, chacune des façons par lesquelles KNitTel transmute la géographie actuelle en fiction apparaît tout à fait simple. Cependant, le processus total est complexe comme le démontre sa Via Mala, c'est-à-dire la route d'Andruss au col de l'Yzolla, soit le Val Medel, de Disentis au Lukmanier. Les localisations déménagées, les éléments principaux omis, la réalité mélangée avec la fiction sont illustrés nulle part mieux que dans ce Val Medel/Via Mala (Figure 2). Essentiellement, KNITTEL combine la géographie actuelle du Val Medel avec l'aura historique de la 
vraie Via Mala. Il mélange l'essence des deux lieux et en fait une coloration intégrée par la capacité de son imagination. Aussi, à partir des repères topographiques réels déjà signalés, il convient, par déduction logique, de replacer dans leur vrai contexte les lieux faussement fictifs. Remontons donc la vallée du Rhin de Medel, depuis Disentis jusqu'au Lukmanier $(20 \mathrm{~km})$.

Par des «flashbacks» fréquents dans son roman, KNitTEl nous parle de la localité de Nauders comme étant le peuplement principal de sa Via Mala (p. 14, 27, 32, 101, 134): une localité formée de plusieurs villages (p. 374) avec son calvaire, qui n'est pas à l'abri des avalanches et dont l'habitat central est haut perché au bord d'un plateau. Il n'y a plus de doute possible: le Nauders de KnitTel, c'est Curaglia, principale fraction de la commune de Medel (Lucmagn). Mais l'écrivain réussit presque à égarer son lecteur car il emprunte un vrai toponyme à la périphérie des Grisons. Nauders est, en effet, un village du Tyrol autrichien, logé à $1394 \mathrm{~m}$ d'altitude, au triplex confinum où convergent la Suisse, l'Autriche et l'Italie, à quatre kilomètres au Nord du Reschenpass (frontière Italie-Autriche).

Puis, toujours en montant vers le haut de la vallée, voici le lieu-dit Yeff avec la maison Imyeff abritant la famille Lauretz. Pour ce lieu fondamental dans son roman, KNITTEL peaufine la précision géographique: «... Yeff entre les villages de Nauders et l'auberge de l'Yzolla, dans le district d'Andruss" (p. 374). Il situe ce lieu-dit assez loin en contrebas du glacier de Medel (p.450) entre Nauders et la chapelle Saint-Hubert (p. 301) et sur l'adret de la vallée (p.8): «... la maison Imyeff... située à une centaine de mètres de la grande route, reléguée dans un coin sous la muraille grise et nue d'une montagne qui la surplombait d'une hauteur de sept cents mètres. La maison à trois étages était étroite, faite de blocs de granit gris, et son toit était calé par de larges pierres» (p. 7). Or, comme KNITTEL ajoute que la maison est toute proche de la cascade mugissante de l'Yzolla (Fumatschfall), la réalité, alors, éclate au grand jour: Yeff, c'est $A$ cla, fraction la plus élevée en altitude de Medel, jusqu'où la route demeure ouverte l'hiver; ce point particulier étant d'ailleurs explicitement confirmé dans le roman. A cela vient s'ajouter un autre argument: Yeff est la déformation de Hof qui, en romanche, se dit Acla (la ferme). Enfin, le romancier situe Yeff à trois heures de marche du col (Lukmanier), ce qui est plausible (p. 47).

En continuant le parcours de montée vers le col, voici la chapelle Saint-Hubert (p.48). «Là, sur un espace herbu projeté du flanc de la montagne, se dressait la chapelle Saint-Hubert avec le bulbe de cuivre de son petit clocher et sa croix d'or brillant au soleil qui rayonnait sur ce coin isolé par dessus l'épaule de glace du pic Cristallina» (p. 122). Ce tableau exact ne permet plus de se tromper: la chapelle Saint-Hubert, c'est Sogn Gagl. Enfin, à l'arrivée au col, KNitTel y place l'auberge Gumpers avec trois indices intéressants: le troupeau de vaches des Gumpers pâturant sur les pentes du Rondadura (p.49), la desserte du col par l'autocar postal (p.49, 50), l'auberge elle-même enfouie complètement sous la neige pendant tout l'hiver (p. 51). L'auberge Gumpers, c'est l'Hospice du Col. Tout près de là, KNITTEL dispose le chalet Lauters (p. 374): à moins d'une demi-heure de marche du col (p. 52), et regardant plein Est vers le Pic Vallatscha (p. 58), ce chalet se situerait donc entre le col proprement dit et les premières pentes du Pic Rondadura.

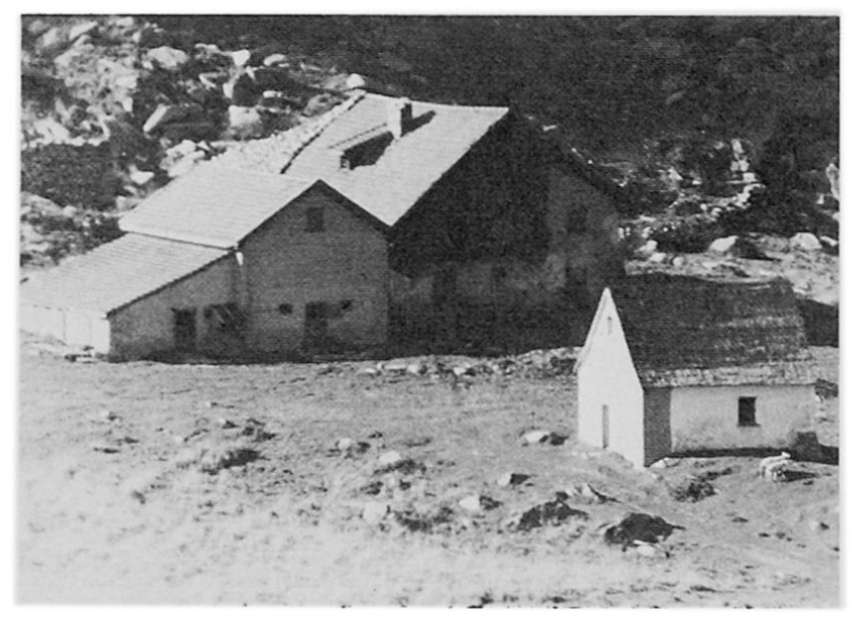

Fig. 6 La chapelle Saint-Hubert

"Là, sur un espace herbu projeté du flanc de la montagne, se dressait la chapelle Saint-Hubert avec le bulbe de cuivre de son petit clocher et sa croix d'or brillant au soleil qui rayonnait sur ce coin isolé par dessus l'épaule de glace du pic Cristallina."

La confrontation entre la description de Knittel et la réalité n'apparaît pas évidente. La chapelle Saint-Hubert de Knittel est celle de Saint-Gall (Sogn Gagl).

L'écrivain place le grand col alpin du Lukmanier (Yzolla) à cinq heures de marche à pied depuis Disentis, ce qui est tout à fait concevable pour 20 kilomètres de montée sur 850 mètres de dénivellation total (p.398). Enfin, si le col de l'Yzolla revient fréquemment sous la plume de Knitrel, il est toutefois surprenant de constater qu'il n'en donne rigoureusement aucune description détaillée et substantielle.

\section{Epilogue}

Aujourd'hui, les voyageurs qui parcourent l'autoroute N 13 Bodan-Bellinzona passent par la véritable Via Mala. Ils sont à mille lieues de penser que le titre du roman de KNITTEL n'a rien à voir avec ce lieu célèbre. En se sens, les touristes visitant la contrée de la Via Mala historique pour expérimenter la saveur de 
l'ouvrage de JoHN KNITTEL seront probablement déçus. Pour l'estivant dans l'Oberland, rien n'indique que la région de Disentis et le Val Medel sont au cœur même de l'intrigue romanesque de cette œuvre mondialement connue. Plus d'une décennie après la mort de KNITTEL, aucun panonceau public n'avertit que Disentis est Andruss, que le Lukmanier est le col de l'Yzolla et que Sogn Gagl est la chapelle SaintHubert. Les familles Lorez, Donatsch, Spescha et CAFLisCH savent-elles qu'elles ont été popularisées en Lauretz, Bonatsch, Specha et Cabflish?

Durant les quarante dernières années, des changements radicaux d'ordre social, économique et spatial ont affecté la Surselva et ses vallées latérales. Plus particulièrement, le Val Medel a été le théâtre de transformations environnementales importantes. En 1964-1967, l'édification du barrage de Santa Maria (hauteur $117 \mathrm{~m}$ et largeur $560 \mathrm{~m}$ ) est venue complètement ennoyer la haute combe alpestre du Lukmanier, à huit mètres sous l'altitude sommitale du col $(1916 \mathrm{~m})$. De ce fait, l'auberge Gumpers, le chalet Lauters et le lieu-dit Santa Maria ont disparu. En avril 1975, Acla a été enseveli sous une avalanche tuant trois habitants et est ainsi pratiquement rayé de la carte. Aussi n'y a-t-il plus de maison Imyeff. Toutefois, une filiation demeure entre le tableau brossé par KNITTEL en 1939 et la situation actuelle: le Val Medel est le type même de la cellule montagnarde où le respect des coutumes et des pratiques n'est pas un vain mot. En ce dernier quart du $\mathrm{XX}^{\mathrm{e}}$ siècle, il offre une image des plus typiques de la haute montagne alpine peuplée (RougIER, p. 122-123).

KNITTEL est mort en 1970 mais la réalité à partir de laquelle il avait bâti sa Via Mala est loin de s'être évanouie. Aussi complets ou aussi fictifs que puissent être les lieux, son décor continue à vivre chaque fois que son roman est lu avec, sans doute, un parfum étrange de nostalgie. Les 560 habitants permanents du Val Medel soupçonnent-ils qu'ils habitent dans la Via Mala de Knittel? En tout cas, pour les lecteurs de Via Mala, quelque part entre Acla et le Lukmanier, les figures charmantes de Sylvelie LAURETZ et ANDi DE RICHENAU continuent à rôder et à exciter notre imagination.

\section{Références bibliographiques}

Knittel, John, Via Mala, Paris, Albin Michel, 1941, version française. Dans l'article présenté ci-dessus, les références à la pagination du roman proviennent du texte publié dans la Collection Le Livre de Poche, $\mathrm{n}^{0} 331$, édition $3^{\mathrm{e}}$ trimestre 1979 (Paris).

Rougier, Henri, Les hautes vallées du Rhin (Grisons), étude de géographie régionale, Gap, Editions Ophrys, 1980 (en particulier les pages 120 à 123 et 288 à 290 consacrées au Val Medel).
HANTKE René: Eiszeitalter, Die jüngste Erdgeschichte der Schweiz und ihrer Nachbargebiete. Band 2: Letzte Warmzeiten, Würm-Eiszeit, Eisabbau und Nacheiszeit der Alpen-Nordseite. 703 S., 273 Fig. und Karten, 1980, Ott Verlag Thun, Fr. 78.--.

Der zweite Band dieses Standardwerkes über das schweizerische und alpine Quartär übertrifft an Gründlichkeit seinen Vorgänger. Das vorhandene Wissen über diesen Gegenstand ist praktisch lückenlos zusammengetragen und vom Autor zu einer Gesamtschau über die eizeitlichen Vorgänge in diesem pleistozänen Schlüsselgebiet aufgebaut worden. Nicht, dass nun alle offenen Fragen beantwortet wären! Die Basis ist indessen geschaffen, von der aus eine weitere Generation von Quartärforschern zielstrebig arbeiten kann. In welcher Gegend sie auch immer ansetzen, das Nachschlagwerk, das ihnen dort den heutigen
Stand der Kenntnisse vermittelt, ist vorhanden. Ein bis in weite Lokaldetails vorstossendes Register erschliesst den reichen Fundus dieses Wissens vorzüglich. Die genaue Beschreibung der Lokalitäten lässt das Werk auch für Zwecke des Landschaftsschutzes verwenden. Die Argumente für eine Unterschutzstellung dieses oder jenes Objektes müssen nun nicht mehr in jedem Einze1falle mühsam erfragt und zusammengetragen werden.

Dank der Unterstützung zahlreicher Institutionen, Behörden und privater Gönner ist das Werk äusserst preiswert erhältlich. Der Autor hat seine immense Arbeit im Frondienst geleistet, was doch ein so kennzeichnendes wie bedenkliches Licht auf die Art wirft, wie hierzulande wissenschaftlich publiziert werden muss.

GH $1 / 82$

Walter Kyburz, Zürich 